\title{
Experiencias
}

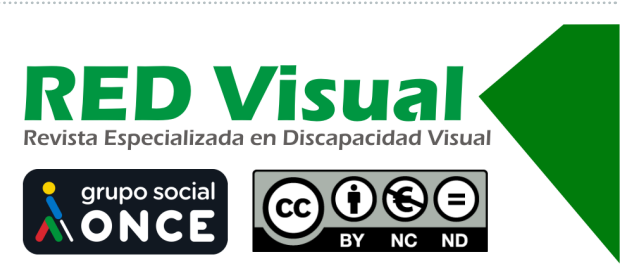

Recepción: 16-12-2020 Aceptación: 06-05-2021

\section{Juegos para Eva: recursos inclusivos cuando un alumno con ceguera participa en un juego motriz ${ }^{1}$}

\section{Games for Eva. Inclusive resources when a visually impaired learner participates in a motor game}

\author{
D. Huguet Mora, A. Blanco Rodríguez, A. Agustina Arias
}

\section{Resumen}

Desde el Centro de Recursos Educativos de la ONCE en Barcelona se ha creado un nuevo recurso dividido en dos bloques como resultado del asesoramiento realizado a los especialistas de educación física de numerosos centros educativos, deportivos y de tiempo libre. El primero consiste en una serie de conceptos sobre la discapacidad visual y unos criterios generales de adaptación que facilitan la comprensión de los recursos inclusivos y ayudan a que los docentes ajusten estos recursos a su realidad y a las diversas variables que intervienen en los juegos. El segundo ofrece, a modo de ejemplo, 21 juegos adaptados con la mayoría de los recursos inclusivos del primer apartado. Se han distribuido los recursos en los diferentes juegos para ofrecer una visión general de las posibilidades de adaptación. Es importante tener en cuenta que cada uno de estos recursos podrían utilizarse en otros juegos; así pues, no hay solo una posibilidad de adaptación, sino diversas estrategias a utilizar en función de las variables que nos encontremos. En definitiva, con los recursos que se incluyen en los 21 juegos de ejemplo se podría adaptar cualquier juego motriz. Nuestro trabajo no es una mera recopilación de juegos adaptados, sino de un documento que permitirá aprender a utilizar recursos verdaderamente inclusivos, ya testados, que facilitarán la inclusión del alumnado con ceguera en los juegos motrices y que se podrán utilizar en la adaptación de cualquier otro juego.

1 Trabajo galardonado con un accésit en la categoría General del v Concurso de Experiencias de Innovación y Buenas Prácticas en Servicios Sociales de la ONCE, presentado bajo el título Juegos para Eva.

Huguet, D., Blanco, A., y Agustina, A. (2021). Juegos para Eva: recursos inclusivos cuando un alumno con ceguera participa en un juego motriz. RED Visual: Revista Especializada en Discapacidad Visual, 78, 83-101. https://doi.org/10.53094/YAMM9031. 


\title{
Palabras clave
}

Juegos motrices. Inclusión. Educación física. Adaptaciones. Centros educativos. Alumnado con ceguera.

\begin{abstract}
The ONCE Educational Resources Centre in Barcelona has created a new resource divided into two blocks as a result of the advice provided to physical education specialists in numerous educational, sports and leisure centres. The first consists of a number of concepts about visual impairment and general adaptation criteria that facilitate understanding of inclusive resources and help teachers to adjust these resources to their own situations and to the different variables that the games involve. The second offers, by way of example, 21 adapted games with most of the inclusive resources of the first area. Resources have been distributed across the games to give an overview of the possibilities for adaptation. It is important to bear in mind that each of these resources could be used in other games; thus, there is not only one adaptation, but various strategies to be used depending on the variables we encounter. In short, with the resources included in the 21 example games, any motor game could be adapted. Our work is not a mere compilation of adapted games but a document that will enable learning about how to use truly inclusive resources which have already been tested, which will facilitate the inclusion of visually impaired learners in motor games and which can be used in adapting any other game.
\end{abstract}

\section{Key words}

Motor games. Inclusion. Physical education. Adaptation. Educational establishments. Visually impaired learners.

\section{Presentación y justificación}

Las sucesivas leyes educativas han ido teniendo una inequívoca vocación hacia la inclusión, por lo que nuestra sociedad ha tenido que ir evolucionando en este sentido. Sin embargo, ¿cómo hacer que se cumpla una verdadera inclusión, concretamente en la práctica de la actividad física?

La realidad de los centros educativos en la actualidad, las ratios profesor/alumno, la diversidad del alumnado, los prejuicios, la falta de conocimientos sobre las capacidades

Huguet, D., Blanco, A., y Agustina, A. (2021). Juegos para Eva: recursos inclusivos cuando un alumno con ceguera participa en un juego motriz. RED Visual: Revista Especializada en Discapacidad Visual, 78, 83-101. https://doi.org/10.53094/YAMM9031. 
del alumnado con ceguera y la formación de base de los especialistas de educación física y de deporte son barreras que dificultan su cumplimiento. Por eso, consideramos que conseguir que el alumnado con ceguera incluido en los centros educativos y en las actividades deportivas y de tiempo libre participe en igualdad con sus compañeros sin discapacidad en la actividad física es un gran reto.

Este reto supone hacer propuestas que faciliten la participación en la actividad física del alumnado con ceguera y también del resto de los compañeros.

En este trabajo que presentamos el reto será la inclusión en los juegos motrices.

Los juegos motrices son esenciales para el ser humano en su etapa de desarrollo. Se ponen en marcha los esquemas de acción y decisión motriz. Se estimulan las habilidades y destrezas motrices que permiten y desarrollan las capacidades perceptivas, simbólicas, expresivas y creativas en diversos entornos y situaciones.

Con su práctica, se aumenta la adaptabilidad a las diversos procesos mentales y motrices. Estos juegos mejoran la toma de decisiones y, al mismo tiempo, se desarrollan habilidades sociales y emocionales de una forma lúdica y dinámica.

En los juegos, el mensaje pedagógico utiliza un lenguaje directo que es captado por el alumnado dentro de su entorno más próximo y personal de una forma casi inconsciente.

Se aprende mientras se juega, se juega mientras se aprende.

La participación del alumnado con ceguera, en esta parte esencial de las sesiones de educación física y en el tiempo libre, es imprescindible y colaborará de una forma decisiva en su ajuste a la discapacidad al reconocer sus capacidades y superarse al ponerlas en práctica.

Hace muchos años que apoyamos la inclusión educativa en toda nuestra zona de influencia (Aragón, La Rioja, Navarra, Baleares y Cataluña) en el área de la educación física y el deporte con alumnos con discapacidad visual. Durante algunos años, hemos asumido la situación de escuela especial $y$, paulatinamente, nos hemos ido adaptando a los cambios en la ley educativa, primero a la integración y, después, a la inclusión que impera actualmente. Es por ello que, dada la riqueza de experiencias

Huguet, D., Blanco, A., y Agustina, A. (2021). Juegos para Eva: recursos inclusivos cuando un alumno con ceguera participa en un juego motriz. RED Visual: Revista Especializada en Discapacidad Visual, 78, 83-101. https://doi.org/10.53094/YAMM9031. 
que nos aportaba la escuela especial, al mismo tiempo que observábamos la realidad en los centros educativos al realizar los asesoramientos y cursos a los especialistas de educación física, hemos podido crear recursos facilitadores para los docentes de educación física y los profesionales de los centros deportivos y de tiempo libre, a los que podemos llamar recursos inclusivos.

Los recursos inclusivos son aquellos que facilitan la inclusión de las personas con discapacidad, visual en este caso, en un entorno normalizado. Es decir, con otros niños y niñas sin discapacidad.

En los juegos motrices, los recursos inclusivos permiten que el alumno con ceguera pueda participar activamente, con pequeños y casi imperceptibles cambios, para que el resto de alumnado sienta que está jugando al mismo juego que ellos conocen. Un juego está adaptado de forma inclusiva cuando, si lo observamos en su desarrollo, continuamos viendo el juego original y tan solo vemos pequeños cambios que facilitan la participación de los alumnos con discapacidad visual.

Los recursos inclusivos para juegos motrices tienen unas características que debemos seguir como norma:

- Que los recursos que se utilicen estén equilibrados para ajustar la ayuda de forma que le permita progresar en su aprendizaje.

- Que sean aceptados por el resto del alumnado.

- Que incluyan elementos que aumenten la motivación y el seguimiento del juego.

- Que las propuestas no aumenten el trabajo de los docentes, sino que lo faciliten.

En ocasiones, se adaptan los juegos de forma excluyente o se utilizan criterios demasiado proteccionistas, al no conocer las posibilidades motrices reales de las personas con ceguera. También puede adaptarse, pretendiendo ayudar más al alumno con ceguera, utilizando un exceso de recursos que limitan el aprendizaje y les sobrecargan de trabajo. Adaptar de una forma no inclusiva puede provocar un «rechazo inclusor». Entendemos por este concepto cuando, como consecuencia de los cambios que se introducen en los juegos para facilitar su participación, el resto del alumnado rechaza el juego y, como consecuencia, al alumno con discapacidad.

Huguet, D., Blanco, A., y Agustina, A. (2021). Juegos para Eva: recursos inclusivos cuando un alumno con ceguera participa en un juego motriz. RED Visual: Revista Especializada en Discapacidad Visual, 78, 83-101. https://doi.org/10.53094/YAMM9031. 
Como parte de nuestra experiencia docente, hemos llegado a la conclusión de que la opción más aceptada por los profesionales es ofrecerles criterios y recursos facilitadores que les permitan aprender a adaptar los juegos que tienen programados y que dominan perfectamente. De esta forma, dada la gran cantidad de posibilidades de adaptación, el profesional ajustará los recursos que ofrecemos a su realidad.

En la actualidad, hemos decidido dar un paso más y ofrecer una carpeta de recursos de adaptación de los juegos motrices más habituales en las sesiones de educación física, deportivas y de tiempo libre basada en nuestras experiencias.

\section{Objetivos}

Hacia el alumnado con ceguera:

- Conseguir una inclusión educativa normalizada.

- Aumentar su participación en los juegos motrices propuestos en esta área.

- Facilitar la comprensión de los juegos y sus posibilidades motrices.

- Aumentar su motivación hacia la actividad física.

- Facilitar y aumentar la relación social con los compañeros del grupo-clase.

- Aumentar su autoestima y autoconcepto al facilitar su participación.

Hacia el profesorado:

- Crear un recurso sencillo y práctico que aporte a los profesionales una herramienta útil que les ayude en su práctica diaria.

- Facilitar la inclusión en educación física y las actividades deportivas y de tiempo libre en que intervenga el juego como contenido.

- Enseñar a adaptar, de forma inclusiva, los juegos motrices.

Huguet, D., Blanco, A., y Agustina, A. (2021). Juegos para Eva: recursos inclusivos cuando un alumno con ceguera participa en un juego motriz. RED Visual: Revista Especializada en Discapacidad Visual, 78, 83-101. https://doi.org/10.53094/YAMM9031. 
- Ofrecer criterios y recursos que faciliten la inclusión en los juegos de los alumnos con ceguera de cualquier etapa educativa.

- Ofrecer una serie de juegos habituales en las sesiones de educación física, con algunos de los criterios inclusivos para facilitar su aplicación.

- Evitar la exclusión de este alumnado en el área de educación física.

\section{Población destinataria de la experiencia}

Alumnos con ceguera incluidos en los centros educativos y de ocio, más todos sus compañeros, ya que también participan en la actividad y aprenden el significado de la inclusión.

Profesionales de la educación física y el deporte de centros educativos, deportivos y de tiempo libre que tengan un usuario con ceguera en situación de inclusión.

\section{Temporalización y fechas de realización}

- Fase previa de recogida de datos.

- Inicio de la experiencia: inicio del curso 2018-2019 con la programación de la secuenciación del proyecto. Propuestas de adaptaciones de los diferentes juegos y práctica en los cursos de profesores.

- Puesta en práctica: inicio del curso 2019-2020 hasta marzo de 2020. Repartición de los juegos entre los centros educativos y visita a cada centro para la valoración de la idoneidad de las adaptaciones.

- Fase de elaboración escrita: de marzo de 2020 hasta noviembre de 2020.

\section{Metodología}

Utilizamos la metodología de investigación-acción, que es el estudio sistemático orientado a la mejora de la práctica educativa para grupos de sujetos, participando a través

Huguet, D., Blanco, A., y Agustina, A. (2021). Juegos para Eva: recursos inclusivos cuando un alumno con ceguera participa en un juego motriz. RED Visual: Revista Especializada en Discapacidad Visual, 78, 83-101. https://doi.org/10.53094/YAMM9031. 
de sus propias acciones prácticas y la reflexión sobre los efectos de esas acciones. La investigación-acción tiene una serie de características:

- Sirve para la mejora práctica de un proceso educativo.

- Es participativa y colaborativa.

- Los participantes en la práctica aparecen como coinvestigadores.

- Es interpretativa, porque acepta la interpretación de los resultados de los participantes.

- Es crítica, pues busca la mejora práctica de las acciones y actuar como agentes para propiciar un cambio.

Para implementar esta investigación, hemos realizado las siguientes acciones:

- Recogida de los criterios y adaptaciones de juegos a través de la observación de grupos y entrevistas a profesionales implicados.

- Creación de un método de adaptación.

- Propuesta de adaptación de 21 juegos.

- Cada juego se ha puesto en práctica en un centro diferente, por lo que hemos implicado a 21 centros.

- Evaluación de los resultados y modificaciones.

- Presentación de los resultados en la Carpeta de juegos adaptados con el título de La carpeta de Eva.

\section{Descripción del recurso}

El resultado final es la creación de un recurso para distribuirlo a los profesionales que lo demanden tanto de forma telemática, mediante un PDF, como mediante la creación

Huguet, D., Blanco, A., y Agustina, A. (2021). Juegos para Eva: recursos inclusivos cuando un alumno con ceguera participa en un juego motriz. RED Visual: Revista Especializada en Discapacidad Visual, 78, 83-101. https://doi.org/10.53094/YAMM9031. 
de una carpeta física con los juegos plastificados para poder llevarlos y consultar en el espacio de juego.

El recurso está disponible para su descarga en el siguiente enlace: https://drive.google.com/file/d/19U73QkjwWbCwzCBOSDOHPjtk6BId8MHC/view?usp=sharing.

Esta carpeta se ha creado con un lenguaje sencillo y breve, ya que tiene como principal intención que sea de fácil lectura y que aporte recursos prácticos. Además, debe adaptarse a la situación del sistema educativo actual.

Estructura de esta carpeta:

1. Introducción.

2. Consideraciones previas.

3. Criterios de adaptación.

4. Adaptaciones generales.

5. Juegos adaptados.

En los apéndices se incluye parte del contenido del recurso a modo de ejemplo: concretamente, la presentación de Eva, dos adaptaciones generales y dos juegos adaptados.

\section{Conclusiones}

Con este documento hemos recogido la experiencia colectiva de muchos años: por nuestra parte, la tarea de adaptación de los juegos, y, por parte de los centros educativos, la puesta en práctica de los diferentes recursos para validar los resultados. Todos los juegos que están recogidos en el recurso están probados en centros educativos ordinarios con alumnado con ceguera, y la respuesta de los docentes ha sido muy positiva por la gran acogida de nuestras adaptaciones por ser prácticas, sencillas y solucionar a los profesores de educación física su tarea diaria. De momento, los profesionales con los que hemos trabajado nos indican que resultan criterios y recursos muy útiles y prácticos que les facilita desarrollar sus juegos. Paralelamente, un beneficio que vemos en este documento es que soluciona los juegos concretos, y las adaptaciones de estos juegos pueden ser aplicadas a toda la programación de Educación Física de primaria e incluso de secundaria.

Huguet, D., Blanco, A., y Agustina, A. (2021). Juegos para Eva: recursos inclusivos cuando un alumno con ceguera participa en un juego motriz. RED Visual: Revista Especializada en Discapacidad Visual, 78, 83-101. https://doi.org/10.53094/YAMM9031. 
Debemos destacar todavía dos beneficios más: el primero, en el alumnado con discapacidad visual, ya que puede jugar a los mismos juegos que sus compañeros y así formar parte de la sesión de educación física como uno más; el segundo beneficio sería hacia los compañeros de clase, que pueden observar y valorar cómo el alumnado con ceguera puede realizar la actividad física con normalidad y aprender a realizar ciertas ayudas para que esto sea posible.

Para reforzar este retorno de información, ponemos un enlace para que toda persona que utilice este recurso haga una valoración y podamos adecuar este recurso a las necesidades reales: https://forms.gle/UQ9LzCgxpPEhtsVh6.

Nos quedamos con la frase «Se aprende mientras se juega y se juega mientras se aprende» y le añadimos que todo el mundo tiene el derecho a jugar, independientemente de sus capacidades y características: «Jugamos todos».

David Huguet Mora. Especialista de educación física. Centro de Recursos Educativos de la ONCE en Barcelona. Gran Via de les Corts Catalanes, 394; 08015 Barcelona (España). Correo electrónico:dhm@once.es.

Antonio Blanco Rodríguez. Especialista de educación física. Centro de Recursos Educativos de la ONCE en Barcelona. Gran Via de les Corts Catalanes, 394; 08015 Barcelona (España). Correo electrónico: abr@once.es.

Adrià Agustina Arias. Especialista de educación física perteneciente al Departamento de Educación de la Generalitat de Cataluña. Centro de Recursos Educativos de la ONCE en Barcelona. Gran Via de les Corts Catalanes, 394; 08015 Barcelona (España).Correo electrónico: adaa@once.es.

Huguet, D., Blanco, A., y Agustina, A. (2021). Juegos para Eva: recursos inclusivos cuando un alumno con ceguera participa en un juego motriz. RED Visual: Revista Especializada en Discapacidad Visual, 78, 83-101. https://doi.org/10.53094/YAMM9031. 


\section{Apéndice A. Presentación del recurso incluida en la carpeta de juegos para Eva}

\section{Introducción}

La intención de esta carpeta es dar recursos a los profesores de Educación Física, educadores o cualquier profesional que ponga en práctica juegos en los que haya alumnos con ceguera. Desde el Centro de Recursos Educativos para alumnos con discapacidad visual nos gustaría que fuera un documento práctico y que sirviera para todos.

\section{¿Quién es Eva?}

Una niña que está estudiando en una escuela ordinaria de educación primaria. Es la única alumna de la escuela con ceguera. Le gusta mucho la clase de educación física y siempre le dice al profesor que ella lo quiere hacer todo. Tiene un grupo reducido de buenas amigas, pero, en general, tiene buena relación con todos.

Figura 1. Tres dibujos de Eva

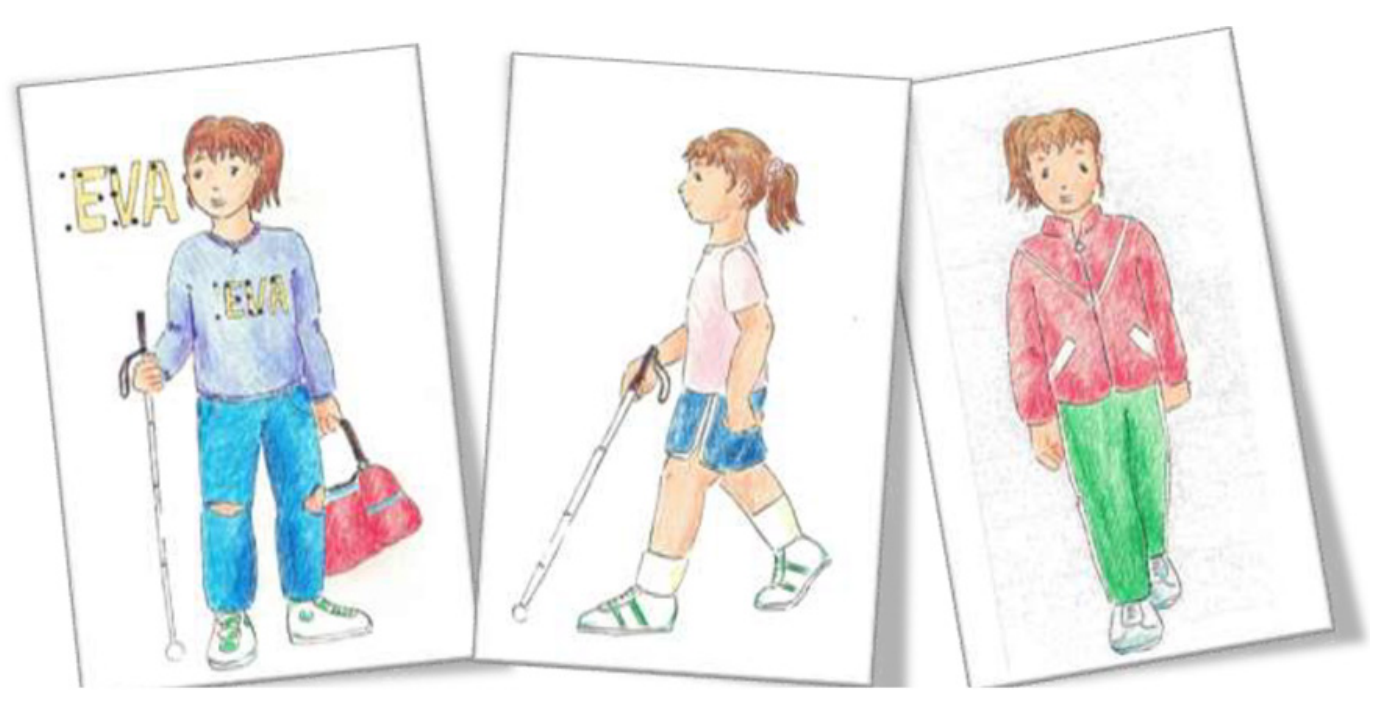

Huguet, D., Blanco, A., y Agustina, A. (2021). Juegos para Eva: recursos inclusivos cuando un alumno con ceguera participa en un juego motriz. RED Visual: Revista Especializada en Discapacidad Visual, 78, 83-101. https://doi.org/10.53094/YAMM9031. 


\section{Apéndice B. Ejemplos de adaptaciones generales incluidas en la carpeta de juegos para Eva}

\section{Adaptación: llamada por la voz}

Es un recurso que utilizaremos en muchos juegos; por ello, haremos una explicación genérica. Cuando una persona con visión hace una carrera, se orienta mirando el punto final hacia donde tiene previsto correr; por ejemplo, si un atleta corre los 100 metros, fija la mirada en la llegada. Para una persona con ceguera, sustituiremos la orientación a través de la visión por la orientación a través del sonido.

- Lo haremos con lo que llamamos «llamada por la voz», que consiste en colocarnos en el punto final de la carrera. Para realizar la llamada por la voz correctamente seguiremos las siguientes pautas:

- Ponemos las dos manos a la boca haciendo como un megáfono.

- Vamos llamando constantemente con palabras rítmicas como: «Va, va, va, va...».

- Cuando el alumno se desvía, quitaremos la mano contraria a la del lado al que la persona se desvía. Si no se desvía, continuamos con las dos manos puestas. Este punto en trayectos cortos no será necesario.

- Si se desvía más, diremos «izquierda» o «derecha» para que corrija. Hay que tener en cuenta que es la derecha o la izquierda de Eva, no de quien Ilama.

- Gritaremos «Stop» si hay cualquier peligro.

\section{Para tener en cuenta}

- Lo que orienta es el punto de voz, lo que decimos no tiene tanta importancia.

- Hay que transmitir seguridad con la voz. Un tono de voz firme y seguro.

- En los juegos, la llamada por la voz la pueden hacer compañeros de clase.

Huguet, D., Blanco, A., y Agustina, A. (2021). Juegos para Eva: recursos inclusivos cuando un alumno con ceguera participa en un juego motriz. RED Visual: Revista Especializada en Discapacidad Visual, 78, 83-101. https://doi.org/10.53094/YAMM9031. 
- Se debe anticipar la llegada, diciendo «vamos llegando» o «poco a poco», y hacer un toque, una vez, en la parte posterior del hombro cuando se ha terminado la carrera. Evitaremos ponernos delante para no chocar.

- Es imprescindible llamar por la voz unos metros antes de una pared u obstáculo que pudiéramos tener detrás, para evitar choques y dar tiempo y espacio para que pueda frenar. Tampoco debe haber niños en la zona de frenado.

Figura 2. Profesor llamando por la voz y Eva corriendo hacia él
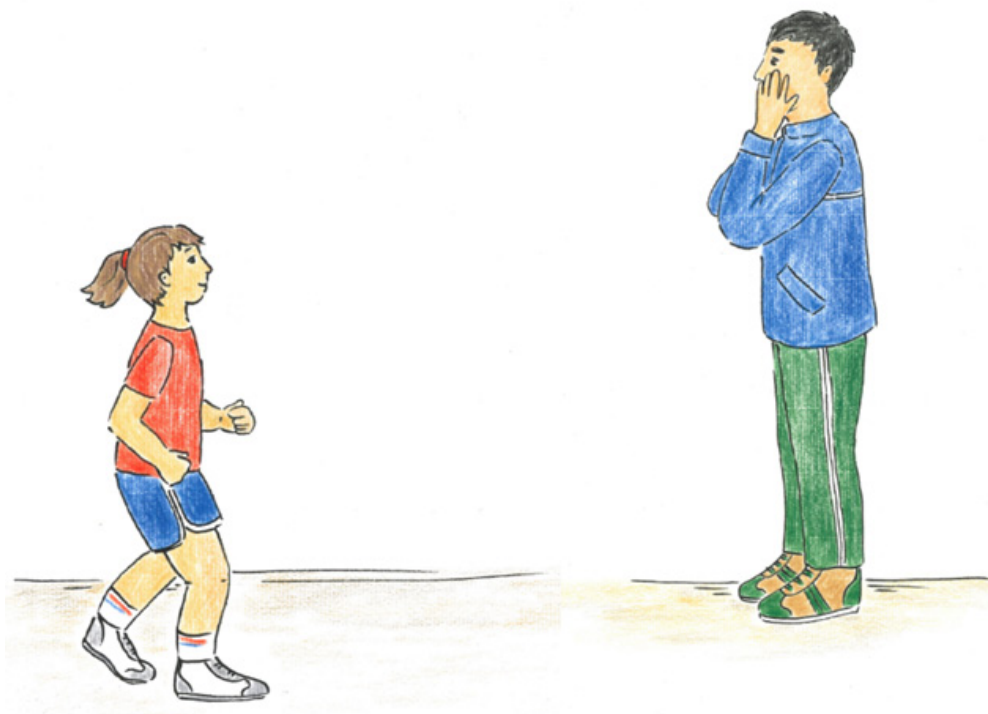

\section{Adaptación: sistema de acompañamiento en carrera}

- Es otro recurso general que utilizaremos cuando no sea posible hacer la llamada por la voz.

- Tal como dice el nombre, habrá un compañero de clase o, a veces, el docente, que hará de guía, tal como se puede observar en las carreras de atletismo en las que participan atletas ciegos.

- La unión entre la persona que guía y Eva será una cuerda, no abrasiva, de unos $45 \mathrm{~cm}$, con sus extremos unidos por un nudo. Se cogen los dos de la cuerda de tal manera que harán la carrera con el movimiento de brazos contrario. En carreras, la cuerda puede ser más larga, pero, en los juegos, la cuerda debe ser más corta para que los 
dos participantes estén muy juntos y poder frenar, girar, cambiar de sentido y todas aquellas acciones del juego.

- Si tenemos los codos en contacto, ayuda mucho a los movimientos y da seguridad a Eva.

- Para aumentar la seguridad, podemos indicar a Eva que lleve el brazo libre doblado por delante.

- Para ayudar a pasar por encima obstáculos o subir a algún lugar, quien guía levanta el brazo.

\section{Para tener en cuenta}

- La persona que guía también puede ir explicando qué pasa en el juego.

- También puede dar instrucciones breves y claras de lo que se va a hacer, como: «nos vamos», «paramos», «frena», etc.

- Procuraremos que el niño guía no sea siempre el mismo; iremos cambiando como si fuera una tarea más del grupo-clase. Cada profesor utilizará sus recursos para que todos los alumnos hagan de guía en algún momento.

- El guía no empuja ni arrastra a Eva, le acompaña al lado.

- La mano libre de Eva va en posición de protección, para aumentar la seguridad en los juegos en dispersión o con muchos participantes.

\section{Beneficios del guía en los juegos}

- La inmunidad del guía: de esta forma, en los juegos de persecución y con balón, el guía puede interponerse y proteger a Eva para facilitarle escapar o que no la toquen. Este recurso, además, puede incentivar la colaboración de los compañeros/ as de clase con Eva.

- Doble opción de jugar: puede ayudar a hacer de guía con Eva, que los compañeros que lo hagan tengan la posibilidad de jugar, después, de nuevo, solos.

Huguet, D., Blanco, A., y Agustina, A. (2021). Juegos para Eva: recursos inclusivos cuando un alumno con ceguera participa en un juego motriz. RED Visual: Revista Especializada en Discapacidad Visual, 78, 83-101. https://doi.org/10.53094/YAMM9031. 
Figura 3. Cuerda de acompañamiento

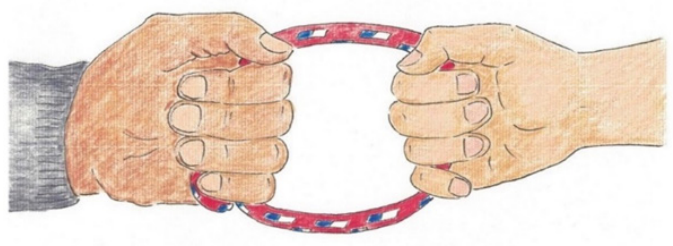

Figura 4. Eva y su guía corriendo con la cuerda de acompañamiento

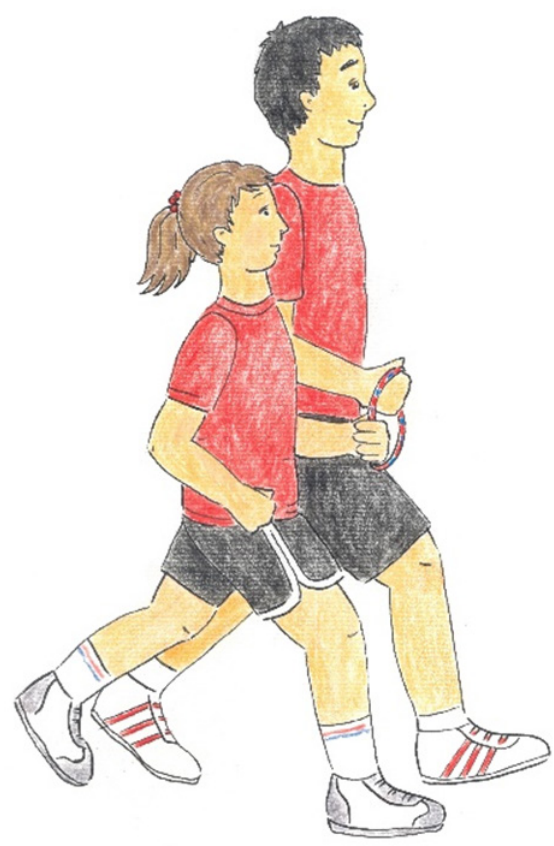

Huguet, D., Blanco, A., y Agustina, A. (2021). Juegos para Eva: recursos inclusivos cuando un alumno con ceguera participa en un juego motriz. RED Visual: Revista Especializada en Discapacidad Visual, 78, 83-101. https://doi.org/10.53094/YAMM9031. 


\section{Apéndice C. Ejemplos de juego incluido en la carpeta de juegos para Eva}

\section{Las jarras (dos sí, tres no)}

Descripción del juego

- Un niño persigue a otro.

- Todo el alumnado se coloca por el espacio en parejas haciendo un círculo grande, cogidos de los codos, como si fueran jarras.

- Si el niño al que persiguen se agarra a una pareja de «jarras», el que está al otro lado del grupo de 3 , sale a perseguir al que está sin pareja.

- Se puede correr por dentro y por fuera del círculo.

- El que perseguía pasa a ser perseguido.

Figura 5. Representación de la posición del alumnado en el terreno de juego

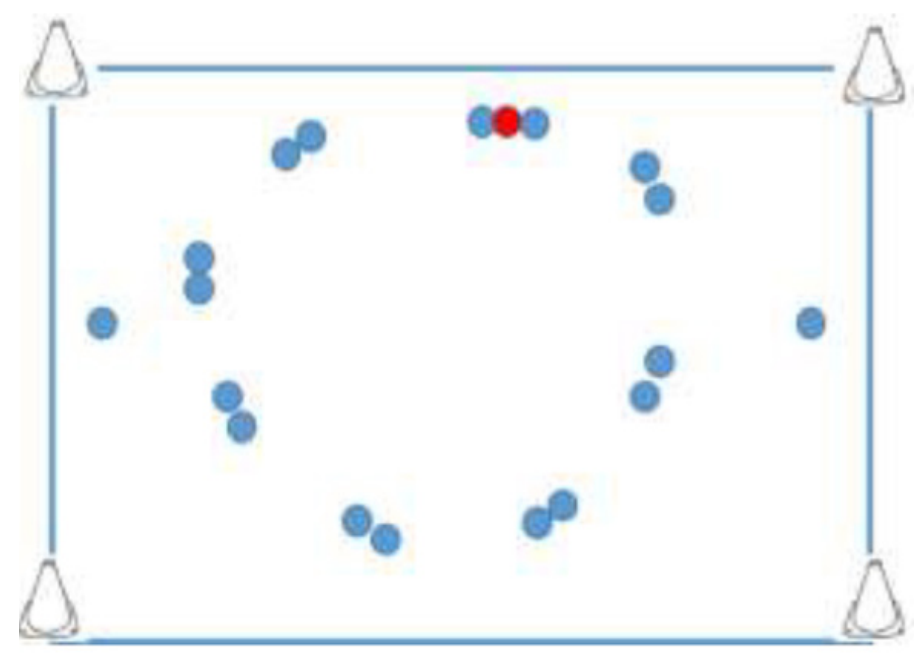

- Si un perseguidor toca a un niño perseguido, cambian los roles: quien perseguía pasa a huir y quien huía pasa a perseguir, pero deberá contar hasta 5 en voz alta antes de empezar a perseguir. 


\section{Adaptaciones para Eva, alumna con ceguera}

- La jarra de Eva será de 3, y ella estará en medio cogida con dos cuerdas de acompañamiento (adaptaciones generales 6), una en cada posible guía.

- Si alguno de los niños perseguidos se agarra a la jarra de 3, Eva y el guía que esté al otro lado irán a perseguir al que está sin pareja. El guía le dice «Vamos» para empezar.

- Eva perseguirá con un guía y las dos cuerdas, una en cada mano.

- Cuando el niño perseguido agarra a una jarra, el niño guía debe decir «Huimos» e ir rápido a agarrarse a una jarra.

- El guía se agarrará a una jarra y se volverán a situar de la siguiente manera: guía-Eva-guía.

El niño guía que esté con Eva irá informando de cómo va el juego: quién está persiguiendo a quién, si pasa alguna anécdota..., entre otras informaciones.

Figura 6. Eva guiada por dos compañeros mientras el perseguidor intenta atrapar a una chica

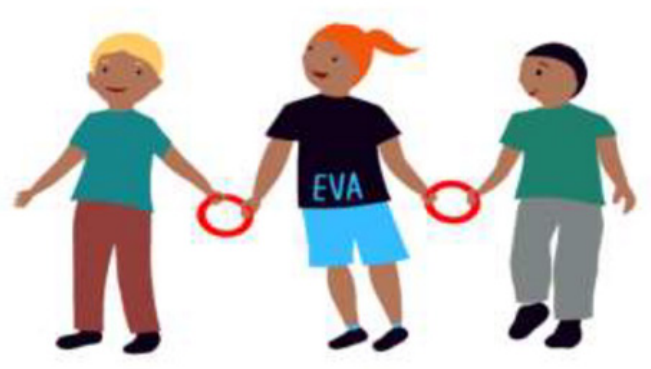

Huguet, D., Blanco, A., y Agustina, A. (2021). Juegos para Eva: recursos inclusivos cuando un alumno con ceguera participa en un juego motriz. RED Visual: Revista Especializada en Discapacidad Visual, 78, 83-101. https://doi.org/10.53094/YAMM9031. 


\section{El pañuelo}

Figura 7. Representación de la posición del alumnado en el terreno de juego

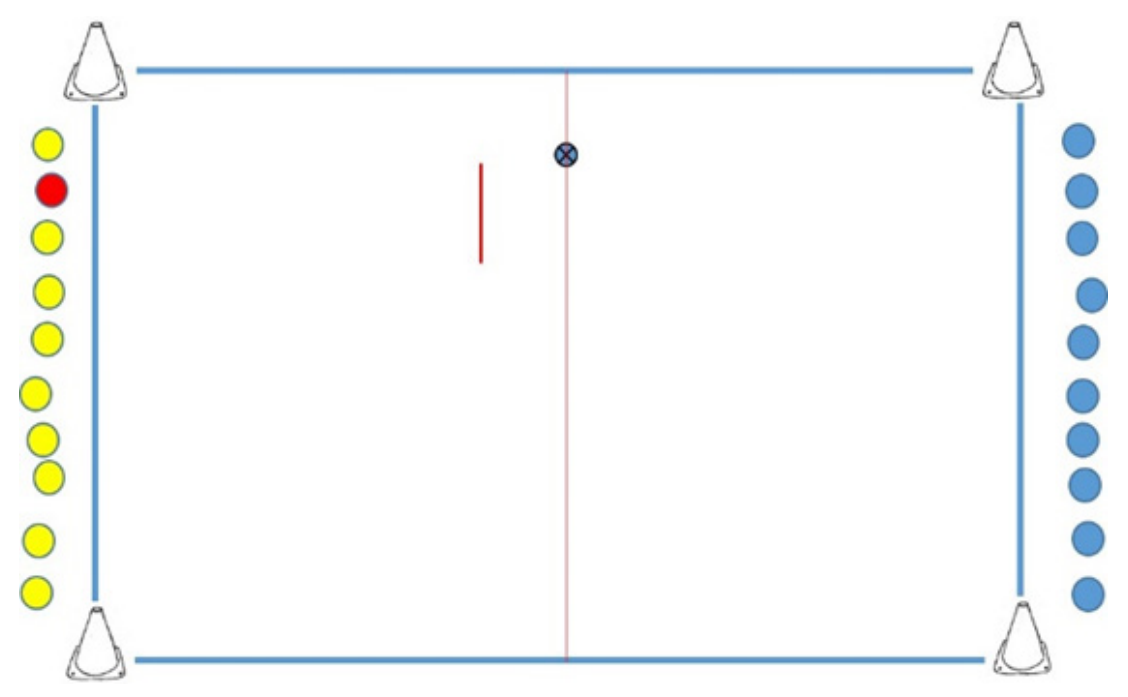

Descripción del juego

- Se marca un rectángulo de juego $(18 \times 9 \mathrm{~m})$.

- Dos equipos enfrentados colocados en ambos extremos del campo.

- En medio del rectángulo y a la misma distancia de los dos grupos se coloca el docente con un pañuelo.

- Todos los participantes de cada equipo se numeran, cada uno será un número.

- El docente dice en voz alta un número de los que tienen los participantes asignados.

- Cada participante que escuche su número debe ir corriendo a atrapar el pañuelo que lleva el docente.

- Se trata de atrapar el pañuelo y llevarlo a su campo.

- El niño que no ha cogido el pañuelo puede atrapar al que tiene el pañuelo.

Huguet, D., Blanco, A., y Agustina, A. (2021). Juegos para Eva: recursos inclusivos cuando un alumno con ceguera participa en un juego motriz. RED Visual: Revista Especializada en Discapacidad Visual, 78, 83-101. https://doi.org/10.53094/YAMM9031. 
- Si lo coge el oponente queda en prisión, si no lo atrapa el que queda en la cárcel es él.

- La prisión se encuentra junto al docente en el lado más cercano a su equipo.

- Cuando un participante agarra el pañuelo y toca a un/a compañero/a que está en la cárcel, este queda liberado.

\section{Adaptaciones para Eva, alumna con ceguera}

- Utilizaremos la llamada por la voz del docente cuando dice el número de Eva (adaptaciones generales 4); el resto de los niños jugará igual, sin adaptaciones.

- Colocaremos una línea táctil (adaptaciones generales 2) en el suelo a 1,5 m del docente por el lado del equipo de Eva.

- El docente mira quién llega antes, si Eva a la línea de $1,5 \mathrm{~m}$ o su oponente al pañuelo, y el docente para el juego diciendo «Stop» para evitar que los dos niños choquen.

- Quien llegue antes se queda el pañuelo con el juego parado.

- Si el pañuelo lo tiene Eva, se pone sobre la línea táctil de 1,5 m de cara a su equipo, y cuando el profesor dice «Ya» empieza a correr hacia su equipo mientras uno de ellos la llama por la voz (adaptaciones generales 4). Su oponente intenta atraparla.

- El niño perseguidor sale desde el medio campo.

- Si el pañuelo no lo coge Eva, el docente hace de guía y la acompaña para atrapar al niño que tiene el pañuelo (adaptaciones generales 5).

- Eva salva a sus compañeros diciendo el nombre en voz alta.

- Cuando tocan a Eva para salvarla, un niño que está en la cárcel le hace de guía para volver a la línea de su equipo y queda directamente salvado.

- El resto de jugadores juega de la forma habitual.

Huguet, D., Blanco, A., y Agustina, A. (2021). Juegos para Eva: recursos inclusivos cuando un alumno con ceguera participa en un juego motriz. RED Visual: Revista Especializada en Discapacidad Visual, 78, 83-101. https://doi.org/10.53094/YAMM9031. 
Figura 8. Eva y su contrincante corren hacia el pañuelo

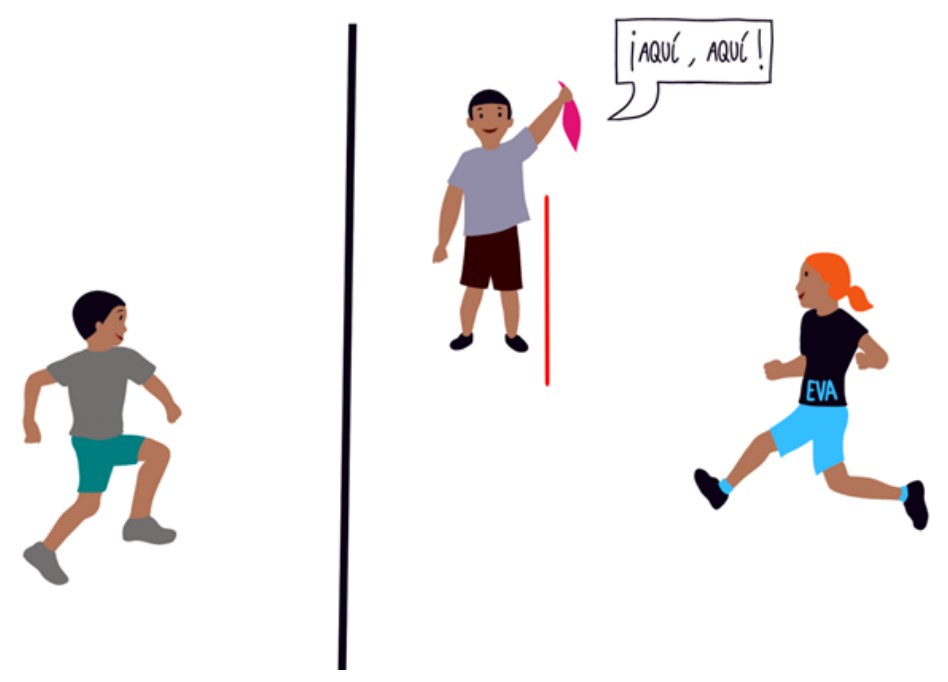

Huguet, D., Blanco, A., y Agustina, A. (2021). Juegos para Eva: recursos inclusivos cuando un alumno con ceguera participa en un juego motriz. RED Visual: Revista Especializada en Discapacidad Visual, 78, 83-101. https://doi.org/10.53094/YAMM9031. 